\title{
Assembly of L-cys coated Quantum Dots-Au@Ag nanorods structure with fluorescence enhancement
}

\author{
Xiaoming Lin, Renming Liu, Jiahua Li, Xuehua Wang* \\ Key Laboratory of Optoelectronic Materials and Technologies, School of Physics and Engineering, \\ Sun Yat-sen University, Guangzhou 510275, China \\ *wangxueh@mail.sysu.edu.cn
}

Keywords: ligand exchange, low-cost, tunable, coupling, plamonic enhancement.

\begin{abstract}
In this paper, Au nanorods and Au@Ag nanorods were synthesized by seed -mediated growth process, and the Colloidal quantum dots CdSe/CdS/ZnS were produced by successive ionlayer absorption and reaction methods. A low-cost and practical ligand exchange of oil phase quantum dots and amino-functionalized Au@Ag nanorods was employed for the coupling process. The surface plasmonic peak is tunable by controllable operation and the photoluminescence peak of quantum dots can be adjusted to corresponding site by successive ion-layer absorption and reaction methods. This operation creates a good condition for plasmonic enhancement. By analysis of the time-resolved fluorescence intensity and the photoluminescence lifetime, we found the energy transfer of this structure. It's a feasible method for coupling the metal nanorods with QDs.
\end{abstract}

\section{Introduction}

The coupling between metal nanorods and quantum dots has attracted great attention in the past decades. When the photoluminescence(PL) peak approaches the localized surface plasmon resonanc(LSPR), great enhancement of fluorescence will be observed. Researches in this area are very popular and widely employed in nano Electronics, optoelectronics, catalysis, biological medicine and so on[1-4]. Preparation, assembly and application were three important aspects in the nano material research. Recently, except for spherical gold nanoparticles which have been widely studied, the anisotropy gold nanoparticles, especially the synthesis, self-assembly and application of Au nanorods[10], has attracted great attention of scientific community. Most of the intersection was localized Surface Plasmon Resonance [5-7].

In the previous work, when coupling between the metal nanorods with quantum dots or fluorescent dyes, fluorescence quenching occurred if two nano particles closely linked together. Therefore, ligand exchange and medium were used. But most of the ligand they used was too expensive for some laboratories. Another, the medium is cheap but not precision adjustable as ligand in coupling process. Mostly, the medium will stick large amount of quantum dots irregularly [8-9].

In this report, we used a simple and practical method to synthesis Au nanorods and silver coated Au nanorods. The colloidal quantum dots $\mathrm{CdSe} / \mathrm{CdS} / \mathrm{ZnS}$ were produced by successive ion-layer absorption and reaction methods. Low-cost and practical chemical(L-cys) was used in ligand exchange of oil phase quantum dots. The Amino-functional nanorods coupled with quantum dots was observed by transmission electron microscope(TEM) image. The assemble structure has six times fluorescence enhancement and be useful for the applications ranging of advanced material. Our flexible method can greatly save the cost, and provides convenience for us to study the effect of plasmon enhancement. Finally, we solved the interpretation problem of fluorescence enhancement effect.

\section{Experimental}

Material. Chloroauric hexadecyltrimethylammonium Hexadecyltrimethylammonium $\begin{array}{cccr}\text { acid(HAuCl4 } & 99.99 \%), \quad \text { Silver } & \text { nitrate(AgNO3), } \\ \text { bromide(CTAB), } & \text { L-Ascorbic } & \operatorname{acid}(\mathrm{AA}), & \mathrm{N}- \\ \text { chloride(CTAC), } & \text { Sodium } & \text { borohydride(NaBH4), } & \text { Cadmium }\end{array}$ 
oxide(99.99\%), selenium, trioctylphosphine(TOP), 1-octadecene(ODE), oleic acid(OA), 1Hexadecanamine(HDA), 11-mercaptoundecanoic acid(MUA), L-Cysteine. All organic solvents was purchased from Aladdin. All chemicals were used without any further purification.

Method. Au nanorods and Au@Ag nanorods were synthesized by seed -mediated growth process in CTAB solutions.

Synthesis of Au nanorods: all reaction of Au nanorods were set at $25^{\circ} \mathrm{C}$ to ensure reproducibility of the subsequent steps. The seed solution was prepared by dissolving $\mathrm{HAuCl} 4(25 \mu \mathrm{l}, 0.1 \mathrm{M})$ in an aqueous solution of $\mathrm{CTAB}(10 \mathrm{ml}, 0.1 \mathrm{M})$. Under vigorous stirring for 1 hour, ice cool NaBH4$(0.6 \mathrm{ml}$, $0.01 \mathrm{M}$ ) was quickly injected into the seed solution. 5 minutes later, the color of the reaction solution became from bright yellow to brown and ready to used for growth solution. Another, the growth solution was prepared by dissolving $\mathrm{HAuCl} 4(25 \mu \mathrm{l}, 0.1 \mathrm{M}), \operatorname{AgNO}(0.08 \mathrm{ml}, 0.1 \mathrm{M})$ and CTAB $(100 \mathrm{ml}, 0.1 \mathrm{M})$ for 30minutes under vigorous stirring. Following, L-Ascorbic acid was added into the solution for 20 minutes to make clear solution. Fllowing, $0.1 \mathrm{ml}$ seed solution was added into the growth solution and the solution was changed from clear to violet in 10minutes, indicating the growth of Au nanorods.

Synthesis of Au@Ag nanorods: as Au nanorods synthesized above was rinsed with deionized water, $2 \mathrm{ml}$ solution of Au nanorods was added into the CTAC solution(2ml, 0.08M). Calculated amounts of $0.01 \mathrm{M}$ AgNO3 and $0.1 \mathrm{M} \mathrm{L}$-Ascorbic was added into the solution above(volume ratio of AgNO3 and L-Ascorbic was 2:1). Following, the mixture was heated to $65^{\circ} \mathrm{C}$ for $3 \sim 5$ hours and the colour of stock solution changed depend on the amount of AgNO3 used before, indicating different size of Au@Ag nanorods.

Colloidal core/shell/shell CdSe/CdS/ZnS QDs were produced by successive ion-layer absorption and reaction methods.

Synthesis of CdSe core: The mixture of $13 \mathrm{mg}$ CdO, 0.3ml OA, 1.056g HAD, 4ml ODE was heated to $120^{\circ} \mathrm{C}$ for 12 minutes under vacuum, Than the solution was heated to $280^{\circ} \mathrm{C}$ under argon. Following, the stock solution, which was mixed by $8 \mathrm{mg}$ Se and $2 \mathrm{ml}$ TOP under vigorous stirring and prepared by the same process as OA-Cd solution, were quickly injected to the hot solution. While the temperature of the growth solution became stable, the growth temperature was reduced to $250^{\circ} \mathrm{C}$ for 1 hour until no significant growth. All the process was under vigorous stirring.

Synthesis of CdS/ZnS shell/shell: the stock solution of Cd was prepared by heating the mixture of CdO, OA, ODE(the molar ratio of CdO to OA was 1:8, and ODE was added to reach the final concentration of $0.1 \mathrm{M}$ ) to $100^{\circ} \mathrm{C}$ for 30 minutes under vacuum. Than the solution was heated to $260^{\circ} \mathrm{C}$ under argon to get a clear solution. The precursors of $\mathrm{S}$ was dissolving element sulphur in ODE to reach concentration $0.1 \mathrm{M}$. The heating process was the same as Cd. All the shell precursors was cooled and used at room temperature. The shell solution of $\mathrm{ZnS}$ were $0.1 \mathrm{M} \mathrm{Zn}$ and $0.1 \mathrm{M} \mathrm{S}$. the process was similarly to the cadminium OA, but CdO was replaced by $\mathrm{Zn}$ acetate, and the solution was heated for $1.5 \mathrm{~h}$ to make clear solution. Each shell was $0.35 \mathrm{~nm}$ through calculation. After each shell growth, the QDs were annealed for $20 \mathrm{~min}$.

Precipitation procedure: separation of unreacted precursors from QDs was performed by extraction followed by precipitation(twice for each process). For extraction, a small amount of toluene and large amount of methanol were added. The phase was easy separated. After centrifugation, the upper phase was discared. After the second extraction, the precipitation was redispersed in toluene.

Coupling of nanoparticals: as syntheisized QDs were redispersed in toluene and nanorods reispersed in 0.1M CTAB, funtionalized process was necessary for coupling . Ligand exchanged of quantum dots were taken in the mixture of $4 \mathrm{mg}$ L-Cysteine in $10 \mathrm{ml}$ water and syntheisized QDs in toluene. Two days later, the layer at the bottom was taken for used. $5 \mathrm{mg}$ 11-mercaptoundecanoic acid was dissolved in $10 \mathrm{ml}$ ethanol and adjusted to $\mathrm{PH} 10$ with $0.1 \mathrm{M} \mathrm{NaOH}$. Nanorods in CTAB was added into MUA solution. After 25minutes, solution of EDC(N-(3-dimethylaminopropyl)-Nethylcarbodiimide hydrochloride) and NHS(N-hydroxysuccinimide) were adjusted to $\mathrm{PH} 5.5$ in MES buffer. Then $500 \mu \mathrm{l}$ EDC and $300 \mu \mathrm{l}$ NHS were added and allowed to react for 1 hour. The activated QDs were then added into the solution. Two days later, precipitation procedure was taken 
to get the coupling products.

\section{Results and Discussion}

\section{The nature of nanoparticles.}

Fig.1(a1) shows the transmission electron microscope(TEM) image of the Au nanods. We found that the shape of Au nanorods was a long strip with its end shaped doom. Its specific size was $60 \mathrm{~nm} * 25 \mathrm{~nm}$. which The larger size of the material can be operated with higher concentration of growth solution. This size of Au nanorods was chosen to coated a layer of silver(fig.1 a2). The ratio of length to width of Au@Ag nanorods is smaller than Au nanorods. Moreover, the shape of Au@Ag nanorods looks like a rectangular in this cross section. Fig.1d shows that the longitudinal and transverse modes of Au@Ag nanorods are near 600nm and 500nm respectively. While the amount of AgNO3 increases, this two modes will blue shift, especially the longitudinal mode. With this method, a certain size of Au@Ag nanorods can be synthesized with calculated amount of reagent. Colloidal quantum dots were produced by successive ion-layer absorption and reaction methods. Fig.1b and fig.1c showed the CdSe(core) and the CdSe/CdS/ZnS(core/shell/shell). Due to the quantum size effect, the photoluminescence peak and UV-vis absorption peak of quantum dots will red shift while the shell grow thicker(as shown in fig.1h). We clearly observed fluorescence of different sizes under 365nm excited light in fig.1c.
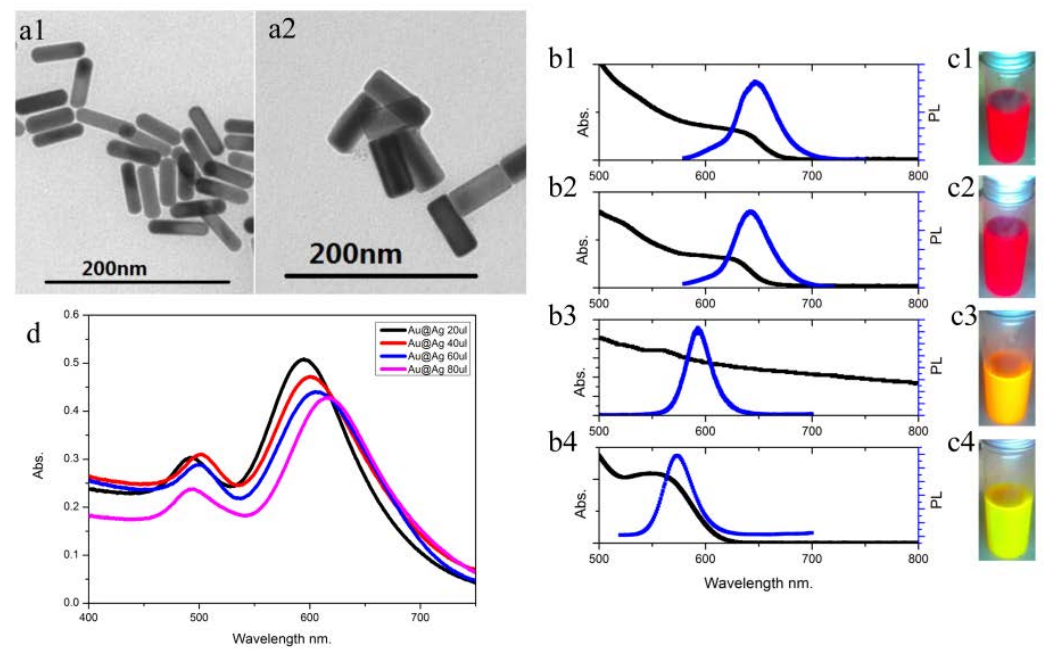

Fig.1.a) transmission electron microscope(TEM) image of the Au nanods(a1) and Au@Ag nanorods(a2). b) the photoluminescence peak and UV-vis absorption peak of different size of quantum dots and c) the fluorescence of different sizes under 365nm excited light. d) absorption

\section{Coupling enhancement.} spectrum of Ag@Au nanorods.

In order to study the enhancement of coupled quantum dots and Au@Ag nanorods, the transmission electron microscopy image was taken to study the structure and the photoluminescence spectrum was used to analyse the PL enhancement(as shown in fig.2). The fabrication of QDs/Au@Ag permits macroscopic production of photophysical processes, such as plasmon-exciton coupling. Fig.2b show that the photoluminescence peak are 597nm. This structure was reacted with CdSe/CdS/ZnS(emission wavelength, 597nm) and Au@Ag nanorods(LSPR peak 600nm). In contrast to the coupling structure, the simple mixture of QDs and Au@Ag nanorods with the same concentration displays no significant enhancement. Mostly because a fraction of aggregated particles didn't separate, the fluorescence quenching was observed in this structure. It's a kind of low-cost and practical ligand that can determine the distance between the QDs and nanorods. The enhancement of fluorescence intensity has increased 6 time. 

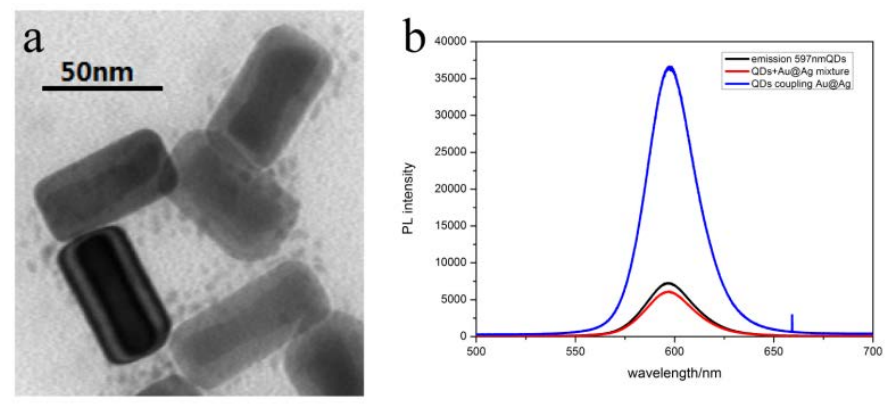

Fig.2 a) transmission electron microscope(TEM) image of Au@Ag nanorods coupled with quantum dots. b) photoluminescence spectrum of QDs(black), mixture of QDs and nanorods(red) and

\section{Exciton dynamics.} coupling structure(blue) with the same concentration.

Although the number of detailed experiment is rather limited, we will still briefly summarize the main exciton dynamics of this structure. At a constant temperature, this reaction exhibited distinct signatures of energetic electron-induced chemical reactions on metals. The transfer of high energy electrons from the metal to reactant initiate the reaction. The exciton lifetime of QDs was considerably decreased from 20.4ns to 5.65ns. This observation of exciton lifetime of quantum dots supports the notion of energytransfer from the QDs to Nu@Ag nanorods. Therefore, we conclude that the energy transfer was enhanced due to the SPR coupling of Au@Ag nanorods with QDs.

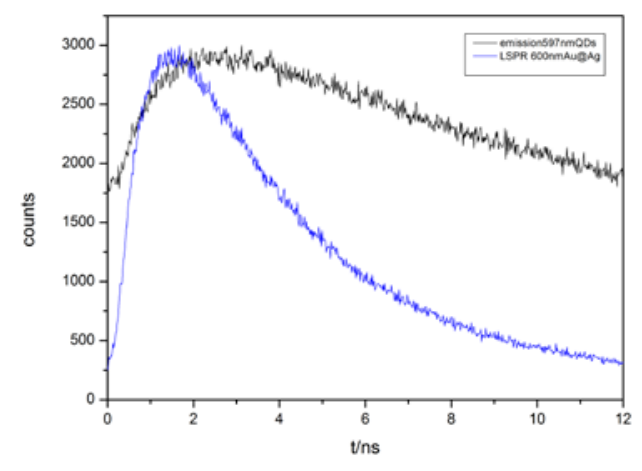

Fig3. Time-resolved PL lifetime QDs(black) and coupling structure(blue).

\section{Conclusions}

In summary, we have demonstrated a kind of low-cost and practical chemical(L-cys) for ligand exchange of QDs. The metallic Au@Ag nanorods coupled with light-emitting CdSe QDs was confirmed by the transmission electron microscope(TEM) image. The PL spectrum of the QDs significantly overlaps with surface Plasmon absorption band of Au@Ag nanorods. Therefore, the absorption energy can be transfer from nanorods to quantum dots, which attribute to the PL enhancement of the structure. The low-cost ligand(L-cys) provides convenience for us to study the effect of plasmonic enhancement. However, the energy transfer rate correspond to the efficiency of quantum yield and the rate of coupling. These are the next problem to be solved.

\section{Acknowledgements}

We gratefully acknowledge the co-workers and tutor Key Laboratory of Optoelectronic Materials and Technologies.

\section{References}

[1] J.H. Bang, P.V. Kamat, ACS Nano 5 (2011),9421.

[2] P.O. Anikeeva, J.E. Halpert, M.G. Bawendi, V. Bulovic, Nano Letter. 9(2009)2532. 
[3] C. Zhang, H. Yeh, M.T. Kuroki, T. Wang. Nat. Mater. 4(2005) 826.

[4] K.E. Sapsford, L. Berti, I.I. Mendintz, Angew. Chem. Int. Ed. 45(2006) 2532.

[5] Navalon, S, de Miguel, M., Martin, R., Alvaro, M. \&Garcia, H. enhancement of the catalytic activity of supported gold nanoparticles for the Fenton reaction by light. J. Am. Chem. Soc.133, 2218-2226(2011).

[6] Chen X., Zhu, H-Y, Zhao, J-G, Zheng, Z-F. \& Gao, X-P. Visible-light-driven oxidation of organic contaminats in air with gold nanoparticle catalysts on oxide supports. Angew. Chemie. Int. Ed. 47, 5353-5356(2008).

[7] Bush, D.G. \& Ho, W. Direct observation of the crossover from single to multiple excitation in femtosecond surface photochemistry. Phy. Rev. Lett. 77, 1338-1341(1996.)

[8] Esteban, Bermudez, Urena, Mark, P, Kreuzer, Stella, Itzhakov. Exciton Enhancement of a Quantum Dot Coupled to a Plasmonic Antenna[J]. Advanced Material, 2012, 24(44): 312-320.

[9] Bishnu, P, Khanal, Anshu, Pandey, Liang, Li. Generlized Synthesis of Hybrid MetalSemiconductor Nanostructures Tunable from the Visible to the Infrared[J]. ACS Nano, 2012, 6(5): 3832-3840.

[10] Suljo, Linic, Pillip, Christopher, David, B, Ingram. Plasmonic-metal nanostructures for efficient conversion of solar to chemical energy [J]. Nature Materials, 2011, 10(12): 911-921. 